\title{
Fibers reinforced composite hydrogels with improved lubrication and load-bearing capacity
}

\author{
Jiawei LI ${ }^{1,2, \uparrow}$, Luyao GAO ${ }^{1,3, \uparrow}$, Rongnian $\mathrm{XU}^{1}$, Shuanhong MA ${ }^{1, *}$, Zhengfeng MA ${ }^{1}$, Yanhua LIU ${ }^{2}$, Yang WU ${ }^{1, *}$, \\ Libang FENG $^{2}$, Meirong CAI ${ }^{1}$, Feng ZHOU ${ }^{1}$ \\ ${ }^{1}$ Lanzhou Institute of Chemical Physics, Chinese Academy of Sciences, Lanzhou 730000, China \\ ${ }^{2}$ School of Mechanical Engineering, Lanzhou Jiaotong University, Lanzhou 730050, China \\ ${ }^{3}$ School of Chemistry and Chemical Engineering, Lanzhou University, Lanzhou 730000, China \\ Received: 19 January 2020 / Revised: 15 March 2020 / Accepted: 22 March 2020 \\ (C) The author(s) 2020.
}

\begin{abstract}
Hydrogels as one kind of soft materials with a typical three-dimensional (3D) hydrophilic network have been getting great attention in the field of biolubrication. However, traditional hydrogels commonly show poor tribology performance under high-load conditions because of their poor mechanical strength and toughness. Herein, pure chemical-crosslinking hydrogels mixed with different types of the micron-scale fibers can meet the requirements of strength and toughness for biolubrication materials, meanwhile the corresponding tribology performance improves significantly. In a typical case, three kinds of reinforcement matrix including needle-punched fibers, alginate fibers, and cottons are separately combined with Poly(n-vinyl pyrrolidone)-poly(2-hydroxyethyl methacrylate (PVP-PHEMA) hydrogels to prepare fibers reinforced composite hydrogels. The experimental results show that the mechanical properties of fibers reinforced composite hydrogels improve greatly comparable with pure PVP-PHEMA hydrogels. Among three kinds of fibers reinforced composite hydrogel, the as-prepared composite hydrogels reinforced with needle-punched fibers possess the best strength, modulus, and anti-tearing properties. Friction tests indicate that the fibers reinforced composite hydrogels demonstrate stable water-lubrication performance comparable with pure PVP-PHEMA hydrogels. Besides, the hydrogel-spunlace fiber samples show the best load-bearing and anti-wear capacities. The improved tribology performance of the composite hydrogels is highly related to mechanical property and the interaction between the fibers and hydrogel network. Finally, spunlace fibers reinforced hydrogel materials with high load-bearing and low friction properties are expected to be used as novel biomimetic lubrication materials.
\end{abstract}

Keywords: cartilage-inspired; fiber-enhanced; composite hydrogel; high strength; good toughness; lubrication; load-bearing; wear-resistance

\section{Introduction}

As one kind of wet/soft polymer-based material with a typical three-dimensional (3D) network, hydrogels possess unique physical and chemical properties, which have got great attention in many fields [1-3]. Meanwhile, the feature of low friction for hydrogels endows hydrogel-based materials with good application potential in the field of biolubrication [4-9]. As reported by Gong et al.[10],

$\dagger$ These authors contributed equally to this work.

* Corresponding authors: Shuanhong MA, E-mail: mashuanhong@licp.cas.cn; Yang WU, E-mail: yangwu@licp.cas.cn 
synthetic hydrogels showed a much lower friction coefficient as low as $10^{-3}$ than hard solid materials. Especially, it is found that the polymer network structure of hydrogels is similar to the extracellular matrix, which plays a crucial role in the friction behavior of cartilage [11-14]. Therefore, hydrogels become ideal materials for artificial cartilage and tissue substitutes. Over the past 20 years, the studies focusing on friction of hydrogels-based materials have achieved great progress. However, the most serious problem of traditional hydrogels is its poor mechanical behavior for high load-bearing. As a result, the development of strong and tough hydrogel materials is the focus of current research [15-18]. For example, researchers have developed a series of hydrogels with high strength and good toughness, such as dual-network (DN) hydrogels [19, 20], nanocomposite hydrogels [21-23], and double-crosslinking hydrogels [24, 25]. Their excellent mechanical properties can be attributed to the effective dissipation of energy by sacrificial bonds introduced in the network during fracture.

Even though many strategies have been developed for improving the mechanical strength of hydrogels, the inherent increase of the strength will shrink the mesh pore size and reduce the degree of surface hydration, and thus leading to the deterioration of surface lubrication property. So, balancing the mechanical properties and hydration capability of the hydrogels becomes a key challenge. Our cartilage simultaneously shows low friction, high strength, and excellent toughness. As been extensively studied, cartilage is considered as a biphasic structure composed of a solid matrix phase and an interstitial fluid phase, in which the interstitial fluid can support most of the contact force to reduce the interface friction, whereas the solid matrix can withstand dynamic loads under shearing. Inspired by this, numerous composite hydrogels with excellent mechanical properties are prepared through combining the fibers and pure hydrogels together, in which the hydrogels can provide a considerable hydration state to reduce the interface friction while the fibers can improve the mechanical property. For example, Huang et al. [26, 27] prepared a series of fibers composite hydrogels. King et al. [28] put forward a new material design strategy, that the hydrogels of the woven fibers were mixed to prepare a new kind of reinforced fibers composite with five times of the strength of steel. However, up to now, enormous efforts devoted to the composite hydrogels mainly focus on the mechanical property, and few cases are focusing on biolubrication. So, developing strong and resilient hydrogels with considerable tribology performance remains a challenge.

As one kind of potential biological material, PHEMA hydrogels are studied extensively because of its good biocompatibility [29, 30]. However, the mechanical strength of pure PHEMA hydrogels is commonly poor and cannot meet the requirements of the cartilage-liked lubrication materials. As mentioned above, the combination of micro-scale fibers and hydrogels can meet both high strength and biocompatibility. Therefore, we prepared fiber reinforced composite hydrogels with both good strength and toughness. In the typical case, microscale fibers (spunlace fibers, alginate fibers, and cottons) are in situ integrated into pure PVP-PHEMA hydrogel network by physical mixing upon encountering radical polymerization. The fibers are robust confined within the hydrogel network through hydrogen bonds. When the normal loads and tangential stress are applied, the fibers can dissipate energy through elongated deformation, thereby improving the mechanical properties of the hydrogels. As a result, the composite hydrogel materials show both high load-bearing and low friction properties, which are expected to be used as novel biomimetic lubrication materials.

\section{Material and methods}

2-hydroxyethyl methacrylate (HEMA) was purchased from J\&K Chemical Ltd. 1-vinyl-2-pyrrolidone was purchased from Shanghai Macklin Biochemical Co., Ltd. Trimethylolpropane trimethacrylate (TMPTA) was purchased from Guangyi Chemical Co., Ltd. 2,2-azobis (isobutyronitrile) (AIBN) was purchased from Tianjin Damao Chemical Reagent Co., Ltd. AIBN was purified by reflux in ethanol. Other reagents and solvents were purchased and used without any purification. Deionized water was applied for all polymerization and treatment processes.

\subsection{Preparation of PVP-PHEMA hydrogels}

The monomer solution was prepared from a mixture 
of HEMA with linear reinforcing polymer- $n$-vinyl pyrrolidone (PVP, 1,300,000 Mw), AIBN $(0.1 \%, w / w)$ as initiator, and TMPTA $(0.5 \%, \mathrm{w} / \mathrm{w})$ as crosslinker. Then the PVP-PHEMA hydrogels were prepared by thermal polymerization of monomer solution at $60{ }^{\circ} \mathrm{C}$ for $4 \mathrm{~h}$. Subsequently, the hydrogel blends were immersed into distilled water for 2 days to remove the residues.

\subsection{Preparation of fibers reinforced composite hydrogels}

Three kinds of woven fabrics made by cotton fibers, spunlace fibers, and alginate fibers were separately used to prepare fibers reinforced composite hydrogels. Firstly, the woven fabrics were immersed in the mixture of monomer solution to allow the complete wettability and liquid saturation for the fiber network. Then the liquid-saturated fabrics were taken out to allow the thermal polymerization for $4 \mathrm{~h}$ at $60{ }^{\circ} \mathrm{C}$ to obtain the fibers reinforced PVP-PHEMA composite hydrogels, as exemplified in the schematic representation. Subsequently, the composite hydrogels were immersed into distilled water for 2 days to remove the residues.

\subsection{Friction test}

The friction test during aqueous-lubricated sliding was carried on the conventional ball-on-disk reciprocating tribometer (CSM Co., Ltd., Switzerland) by acquiring friction coefficient $(\mu)$ at different loads and frequencies. The elastomeric poly(dimethylsiloxane) (PDMS) hemispheres and 314 stainless steel balls with a diameter of $6 \mathrm{~mm}$ were used as pins. The PDMS hemispheres were prepared by a commercial silicone elastomer kit (SYLGARD 184 silicone elastomer, base and curing agents, Dow Corning, Midland, MI) and the weight ratio of the base and curing agents is 10:1. A polystyrene cell culture plate with round-shaped well (Siqi Biotechnology Co., Ltd., Beijing) was used to prepare PDMS hemispheres. After removing the bubbles by vacuum degassing, the mixtures were put into the mold and then incubated in a $70{ }^{\circ} \mathrm{C}$ oven for $4 \mathrm{~h}$. Each sample was measured at least three times at different positions to obtain the average value.

\subsection{Mechanical property measurement}

The mechanical property of the samples was measured by an electrical universal material testing machine with a $500 \mathrm{~N}$ load cell (EZ-Test, SHIMADZU). The test samples were cut into rectangular shape. All mechanical tests were carried out after the sample had reached the swelling equilibrium in water. In the tensile test, the samples were cut into $20 \mathrm{~mm}$ in length, $5 \mathrm{~mm}$ in width, and $2 \mathrm{~mm}$ in thickness. The crosshead velocity was kept at $50 \mathrm{~mm} / \mathrm{min}$ in the tensile measurement. The elastic modulus in the tensile test was calculated from the slope 5\%-15\% of the strain ratio of the stress-strain curve. In the compression test, the samples were cut into $10 \mathrm{~mm}$ in length and width, $3 \mathrm{~mm}$ in thickness, respectively. The compression test was performed at a velocity of $5 \mathrm{~mm} / \mathrm{min}$ with a strain limitation of $70 \%$. The elastic modulus in compression test was calculated from the slope $5 \%-15 \%$ of the strain ratio of the stress-strain curve.

The trouser tearing test was performed in the same machine with a tensile velocity of $50 \mathrm{~mm} / \mathrm{min}$. The samples were firstly cut into a rectangle of 40 $\mathrm{mm}$ in length and $10 \mathrm{~mm}$ in width, respectively. Then the samples were cut into pants in the length direction, and the length of the forked and undivided portions both were $20 \mathrm{~mm}$.

$$
G_{\mathrm{c}}=\frac{1}{t \times L_{\text {bulk }}} \int F \mathrm{~d} \Delta
$$

where the $G_{c}$ represents the energy required to tear. The thickness of the specimen and the length of the tear are indicated by $t$ and $L_{\text {bulk }}$, respectively. $F$ and $\Delta$ stand for load and displacement, respectively (The geometry and dimensions are shown in Ref. [31]).

\subsection{Morphology characterization}

The sample morphologies were characterized by field-emission scanning electron microscope (FESEM) (JSM-6701F, JEOL Inc., Japan) at an accelerating voltage of $5 \mathrm{kV}$. The test samples were prepared according to the standardized process. In a typical case, the test samples were frozen with liquid nitrogen or by an automatic refrigeration system and then were dried in the freeze-drying machine 
for $24 \mathrm{~h}$ at a vacuum degree of $5 \mathrm{~Pa}$ (SCIENTZ-10N, Ningbo Scienzt Biotechnology Co., Ltd.).

\section{Results and discussion}

\subsection{Synthesis process of composite hydrogels}

The fibers play an important role in the wear resistance of composite gels. According to the previous work, PVP-PHEMA gel was capable of achieving a low friction coefficient ( 0.03) [32]. Upon integrating fibers into hydrogels, the composite hydrogels can both realize high load-bearing and low friction. Figure 1 shows the schematic illustration of the fabrication process for the composite hydrogels. First, the mixture containing monomers of HEMA and NVP, initiator of AIBN, and crosslinker of TMPTA was poured into the mold, and then the fiber sheet was added to allow it completely wetted. Finally, the samples were put into $60{ }^{\circ} \mathrm{C}$ oven for $4 \mathrm{~h}$ to perform radical polymerization. After polymerization, the samples were placed into the water to remove the residues. Correspondingly, the fibers penetrate and distribute throughout the entire hydrogel network, and the hydrogen bonds can be formed between fiber surface and polymer chains of hydrogels. So, it can be speculated that the fibers can effectively improve the mechanical strength of hydrogels.

\subsection{Morphology of bare fibers and fiber-enhanced composite hydrogels}

In the hydrogel network, two different kinds of polymer chains (PHEMA and PVP) entangled each other to form a hydrophilic composite network with a large number of hydrogen bonds. As observed from the SEM images, the pristine spunlace fibers exhibit an irregular arrangement with a single fiber diameter of 10-15 $\mu \mathrm{m}$ (Fig. 2(a)), and the alginate fibers show a little regular arrangement with a single fiber diameter of 5-8 $\mu \mathrm{m}$ (Fig. 2(b)), while the cotton fibers show scattered arrangement with single fiber diameter of 15-20 $\mu \mathrm{m}$ (Fig. 2(c)). The formation of a highly porous 3D fiber network ensures the composite hydrogels show high strength and toughness in a different direction. The entire mechanical property of the composite hydrogels must be closely related to the microstructures of the hydrogel network. After freezing-drying cycles, the morphology of hydrogel samples was observed

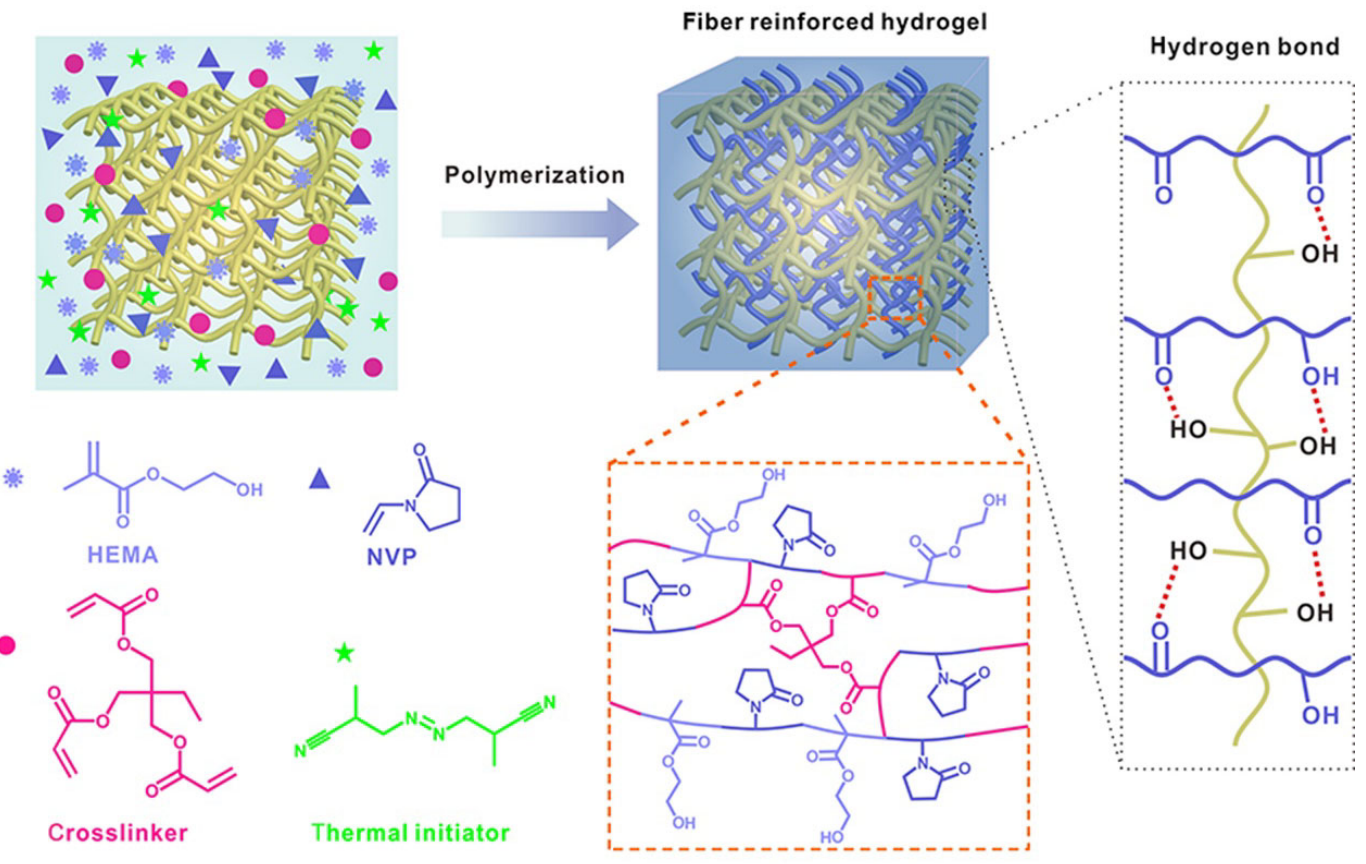

Fig. 1 Schematic illustration showing the preparation process of the fiber-enhanced PVP-PHEMA composite hydrogels along with corresponding molecular structure and network structure, and existing hydrogen interaction between fiber surface and hydrogel network. 

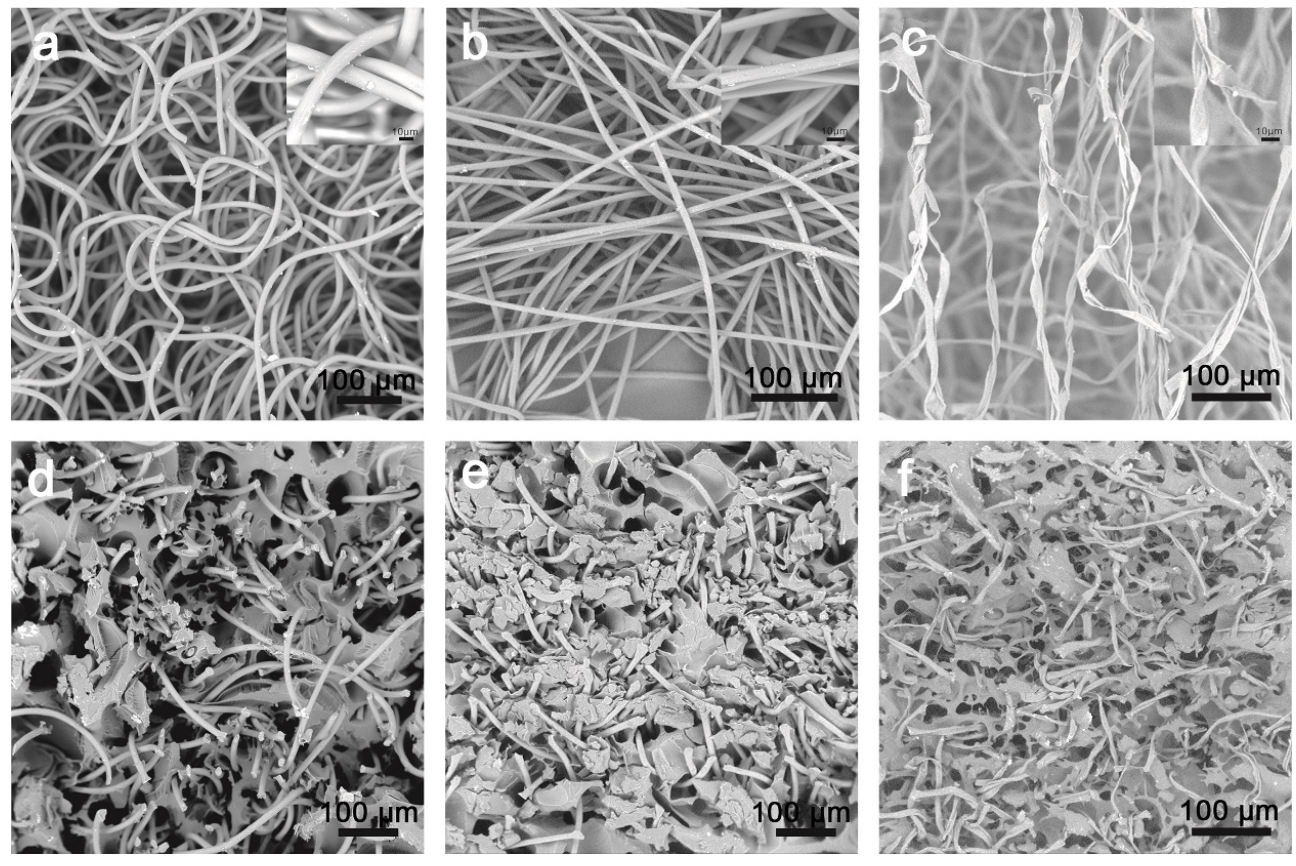

Fig. 2 (a) FESEM images of bare spunlace fibers, (b) alginate fibers, and (c) cotton fibers; network morphologies of (d) spunlace fiber composite hydrogels, (e) alginate fiber composite hydrogels, and (f) cotton fiber composite hydrogels.

by FESEM. The results show that all kinds of fibers were completely encapsulated within hydrogels. In the typical case, after integrating spunlace fibers, the as-prepared composite hydrogels can show a highly porous structure, and the fibers are obvious and irregularly interspersed across the entire samples (Fig. 2(d)). The alginate fiber composite hydrogels (Fig. 2(e)) and the cotton fiber composite gel (Fig. 2(f)) also exhibit similar network distribution structure, but the distribution of cotton fibers within the composite hydrogels is more dispersed than that of the other two fibers.

\subsection{Mechanical property tests for bare fibers and fiber-enhanced composite hydrogels}

Figure 3(a) shows the typical stress-strain curves of pure PVP-PHEMA hydrogels, three kinds of fibers, and fiber-enhanced composite hydrogels. It can be seen that the tensile stress of pure PVP-PHEMA hydrogels is only about $300 \mathrm{KPa}$ at the strain of $4 \%$, which indicates the pure hydrogels is so soft and brittle. The maximum tensile stresses of alginate fibers, cotton fibers, and spunlace fibers are separately $100 \mathrm{KPa}, 1.5 \mathrm{MPa}$, and $3 \mathrm{MPa}$, and their corresponding strains are $49 \%, 55 \%$, and $38 \%$, respectively. Surprisingly, the stress and strain of the hydrogels reinforced with three different kinds of fibers increase sharply. Taking spunlace fiber-enhanced composite hydrogels as an example, the tensile stress of the composite hydrogels can achieve to as high as $9.6 \mathrm{MPa}$, which is $\sim 30$ times higher than pure PVP-PHEMA hydrogels, and the corresponding fracture strain increases to $17 \%$. This indicates that existing fibers can significantly enhance the network density of the hydrogels to endow its good mechanical property. The corresponding elastic modulus shows the same change trend as stress (Fig. 3(b)). The elastic modulus of the pure hydrogels and spunlace fibers are $\sim 1.3$ and $\sim 3.95 \mathrm{MPa}$, respectively. The composite hydrogels reinforced with spunlace fibers can sharply increases to $\sim 28 \mathrm{MPa}$. These data intuitively demonstrate the significant role of fibers within PVP-PHEMA hydrogels. As speculated, the formed compact network of composite hydrogels would effectively shackle the hydrogels and fibers, whereas the fibers break down to dissipate energy. It can be shown that the tensile stress of the spunlace fiber composite hydrogels is the most significant, followed by cotton fibers, and finally the alginate fibers (Fig. $3(\mathrm{a})$ ).

Besides, the excellent mechanical properties of the composite hydrogels were further confirmed by 

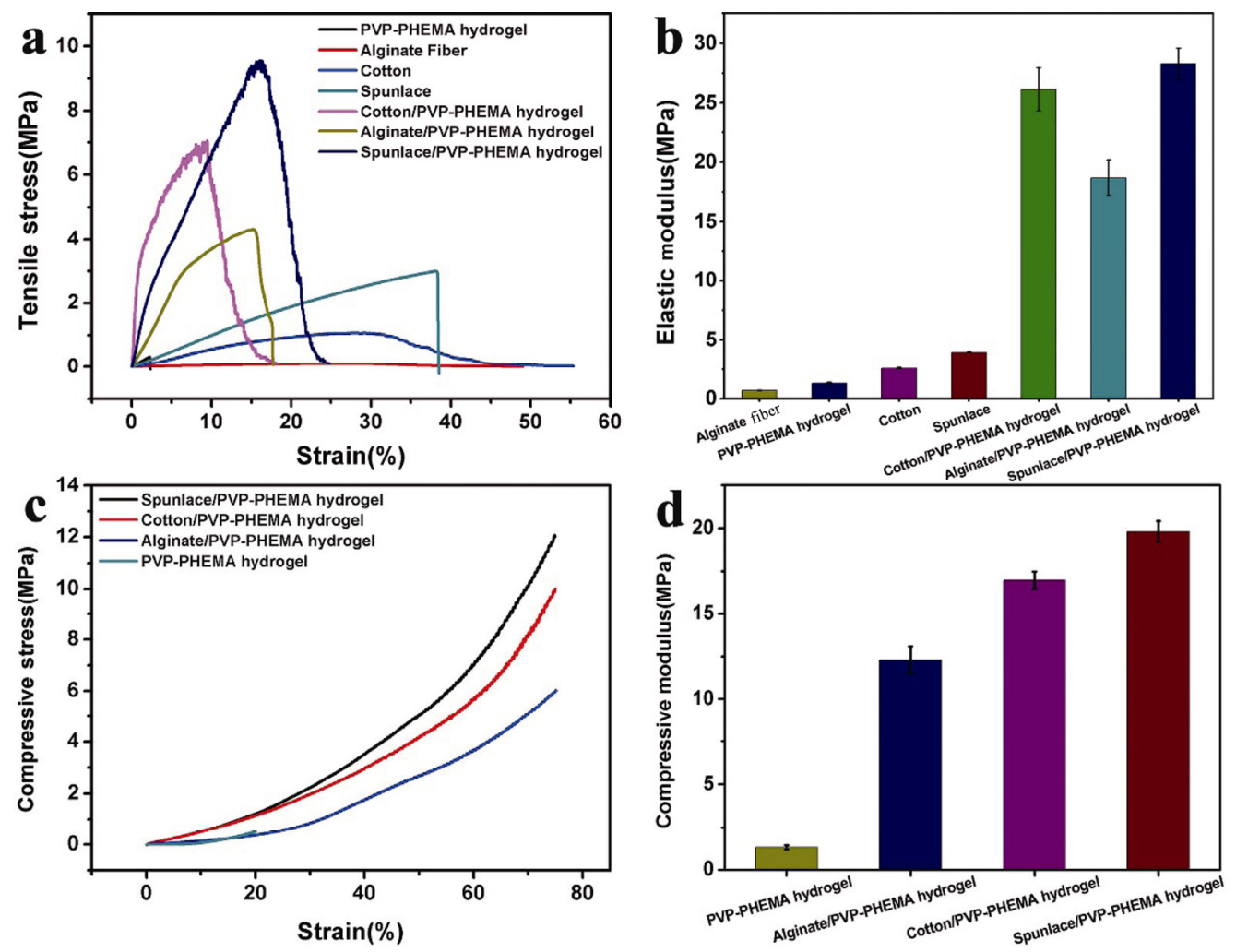

Fig. 3 (a) Tensile stress-strain curves of pure PVP-PHEMA hydrogels, three kinds of bare fibers, and corresponding fiber-enhanced composite hydrogels and (b) the calculated elastic modulus; (c) compression stress-strain curves of pure PVP-PHEMA hydrogels, three kinds of bare fiber and corresponding fibers-enhanced composite hydrogels, and (d) the calculated compressive modulus.

performing compression tests. In terms of spunlace fiber composite hydrogels, it can be compressed to $75 \%$ without fracture and its compression stress can reach $\sim 12 \mathrm{MPa}$ (Fig. 3(c)) and corresponding compressed modulus can reach $20 \mathrm{MPa}$ (Fig. 3(d)). As expected, the excellent anti-compression capacity of composite hydrogels would provide a basic condition to realize high load-bearing under dynamic loading and shearing process. The compression property of the other two kinds of composite hydrogels shows the same trend as that of the spunlace fibers, but relying on the specific feature of each fiber.

\subsection{Tearing test}

The trouser-shape tearing test was used to further verify the toughening mechanism of the composite hydrogels [27]. As shown in Fig. 4(a), the upper end of the hydrogels was clamped to the platform and the other end was fixed to the fixture, and deformed under stress until it was completely torn. Figure 4(b) shows the real-time tear strength- distance curves that are recorded in the tearing test. When the gel is torn along the stretching direction, it first reaches a critical force value than the gel tears following a "zig-zag" tensile stress and exhibits a very long tear distance (Fig. 4(c)). The tear resistance of composite hydrogels is significantly increased compared to pure hydrogel samples. When the spunlace fiber composite hydrogels are torn along the stretching direction, the tear force experiences a rapid increase and then ruptures very rapidly along with short tear distance. This means that the energy required to tear the composite hydrogels is much greater than the energy required for stretching. The maximum tear strength of the spunlace fiber composite hydrogels is $10.1 \mathrm{~N} / \mathrm{mm}$, which is about 25 times higher than pure PVPPHEMA hydrogels $(0.4 \mathrm{~N} / \mathrm{mm})$ and about 10 times higher than bare spunlace fibers $(1 \mathrm{~N} / \mathrm{mm})$. The other two fiber composite hydrogels have lower tear strength than spunlace fiber composite hydrogels (cotton composite hydrogels: $6.8 \mathrm{~N} / \mathrm{mm}$; alginate 

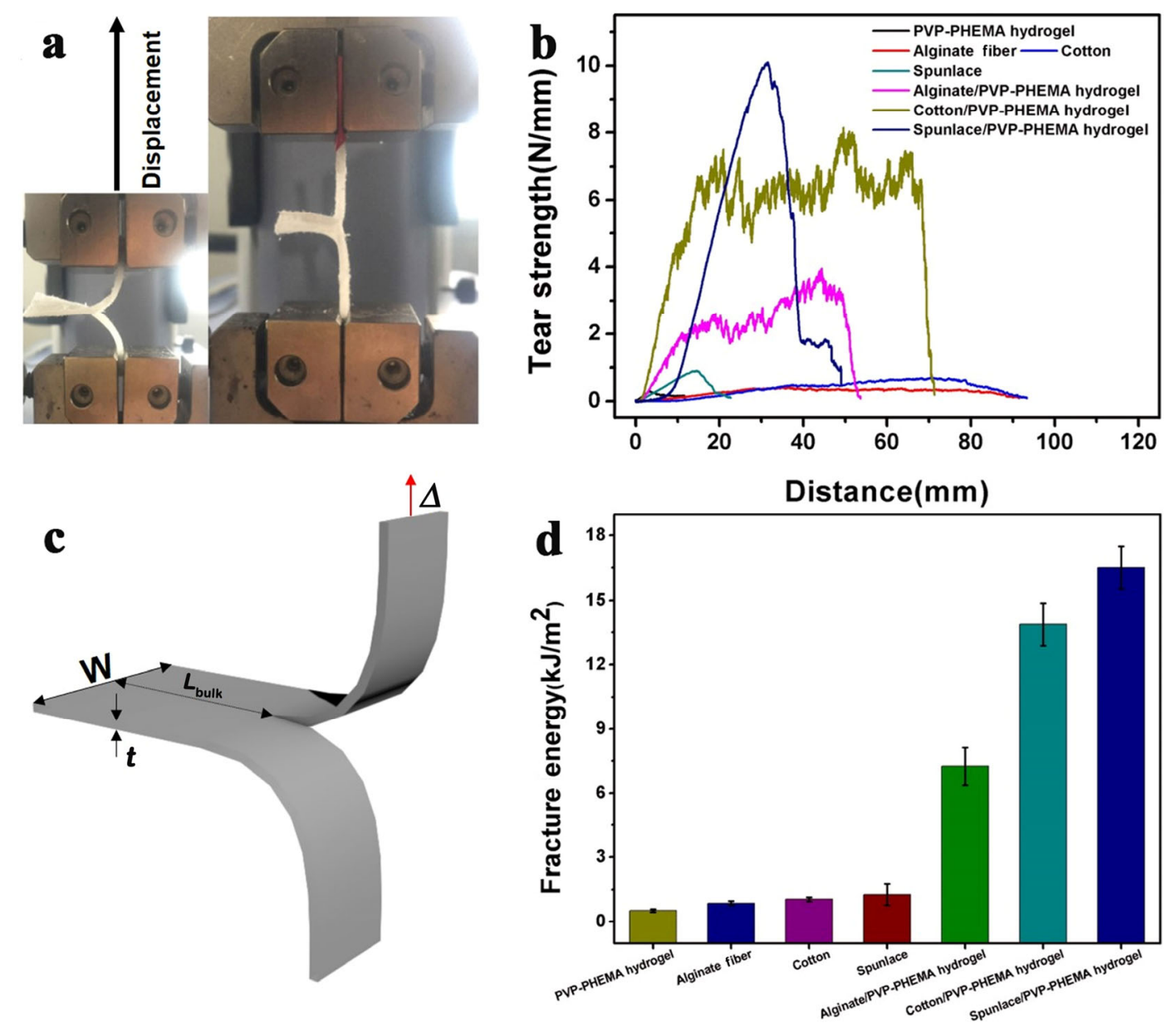

Fig. 4 (a) Photographs of the tearing test for fiber-enhanced composite hydrogels before (left) and (right) after undergoing a large strain deformation; (b) real-time tensile strength-distance curves in tearing tests for pure PVP-PHEMA hydrogels, three kinds of bare fibers and corresponding fibers composite hydrogels; (c) schematic of the tear test and (d) corresponding calculated fracture energy.

fiber composite hydrogels: $2.6 \mathrm{~N} / \mathrm{mm}$ ), but both improved obviously compared to bare fiber samples. What's more, the corresponding fracture energy is shown in Fig. 4(d), which is calculated from the force-distance curves. The fracture energy of the spunlace fiber composite hydrogels can be achieved as $17,000 \mathrm{~J} / \mathrm{m}^{2}$, which is much higher than the $500 \mathrm{~J} / \mathrm{m}^{2}$ of the pure hydrogels. These results indicate that the interaction of the hydrogels matrix with the fiber-reinforced phase promotes synergistic enhancement and toughness to endow the composite hydrogels with excellent mechanical properties. Meanwhile, similar to the tensile strength, the fracture energy of the composite hydrogels also increases as the fiber density of the fiber-reinforced phase increases.

Moreover, to understand the toughening mechanism of the composite hydrogels, the interface morphology of the cracked sample was observed by SEM (Fig. 5(b)). It can be found that during the crack extension process, the fibers inside the hydrogels are gradually stretched, and then occur delayed fracture, which effectively dissipates the energy, so that the hydrogels exhibit excellent tear toughness. Furthermore, at the fracture tip, there is a large amount of intact fibers that can continue to function under a further stretching process until the samples are completely torn (Fig. 5(c)). It should be noted that all three kinds of fibers can form hydrogen bonds with the hydrogel network, but the interaction degree may be slightly different. Therefore, the hydrogen bonds between the fibers and hydrogels play a critical role in the enhancement and toughening of the hydrogels (Fig. 5(a)). 

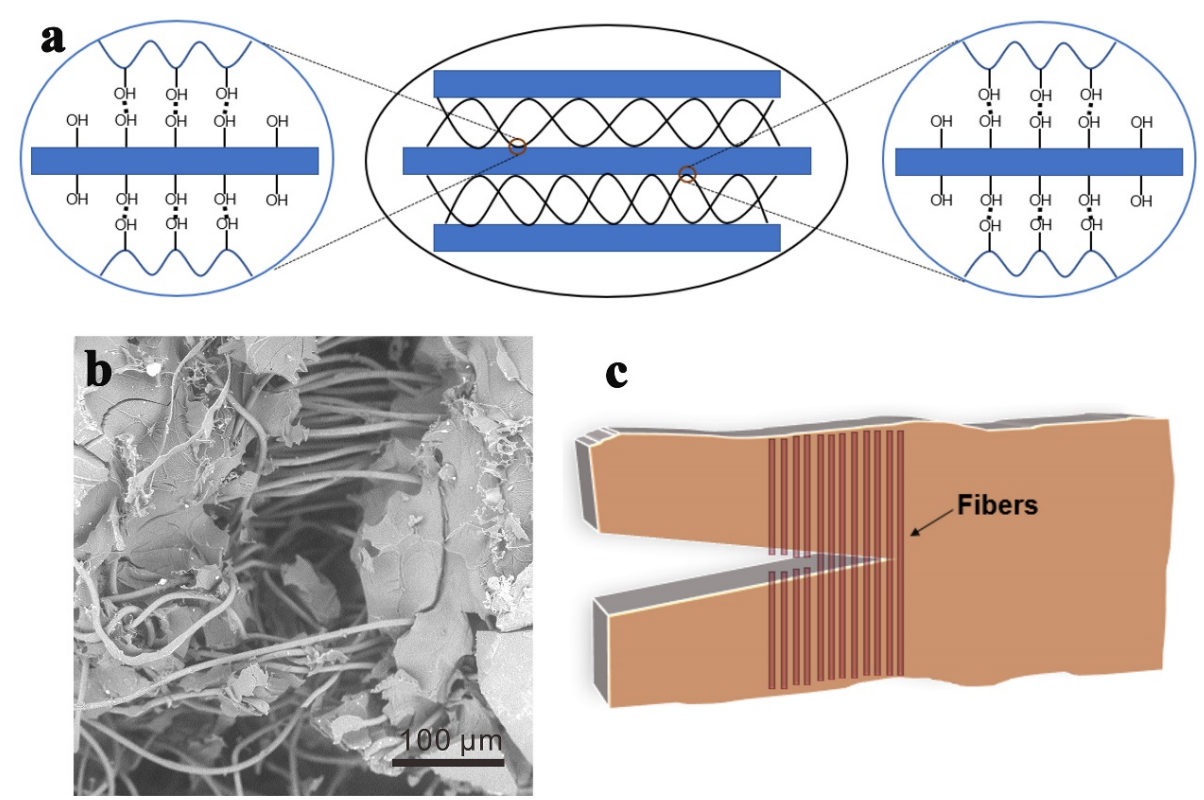

Fig. 5 (a) Schematic diagram of interaction mechanism based on hydrogen bonds between fibers and hydrogel phases; (b) SEM image of the locally interface amplification in tearing tests of spunlace fiber-enhanced PVP-PHEMA hydrogels; (c) the model of delayed-fracture induced energy dissipation mechanism under further stretching tear process.

\subsection{Friction test of fiber-enhanced composite hydrogels}

HEMA and NVP are widely used in the field of contact lenses due to their excellent biocompatibility and good lubricity [33-35]. Figure 6 shows the typical contact image obtained from the sliding process and the corresponding friction curves for pure PVP-PHEMA hydrogels. Friction tests were performed under a ball-on-disk contact style by employing a soft PDMS ball (Young's modulus: $\sim 2.0 \mathrm{MPa}$ ), hard steel ball as sliding pairs, and the deionized water was used as a lubricant. The average friction coefficient was chosen for analysis, which is summarized in Fig. 6(a). It can be seen that the friction coefficient of pure PVA-PHEMA hydrogels against both sliding pairs increased with the normal loads, and the higher friction coefficient was observed when the steel ball was used as a contact counterpart. In the typical case, the friction coefficient is $\sim 0.018$ at the load of $0.2 \mathrm{~N}$ and it increased to $\sim 0.03$ at the load of $2 \mathrm{~N}$ against PDMS pair, and it is $\sim 0.026$ at the load of $0.2 \mathrm{~N}$ and increased to $\sim 0.039$ at the load of $2 \mathrm{~N}$ against steel ball pair, indicating the poor load-bearing property of the pure hydrogels. Correspondingly, the local interface contact process was observed. As shown in Fig. 6(b), the PDMS ball completely penetrated the hydrogel sheet at the applied load of $3 \mathrm{~N}$, resulting from poor mechanical property of the pure PVP-PHEMA hydrogels. The real-time friction coefficient curves were shown under different normal loads against PDMS (Fig. 6(c)) and steel ball (Fig. 6(d)) as sliding pairs. At the initial stage of friction, the sliding interface showed a stable low friction coefficient, but it appeared a rapid increase of the coefficient due to complete broken of hydrogels. This indicates that low mechanical strength of pure PVP-PHEMA hydrogels is not enough for high load-bearing.

The friction coefficients of the spunlace fiber composite hydrogels under different normal loads upon employing PDMS ball and steel ball as sliding pairs are shown in Fig. 7(a). The composite hydrogels exhibit low friction coefficients $(<0.06)$ under a wide range of load from 0.2 to $5 \mathrm{~N}$ both against two kinds of sliding pairs. Compared with pure hydrogels (Figs. 6(c) and 6(d)), the load-bearing capacity of the spunlace fiber composite hydrogels improves highly. In the typical case, the friction coefficient of the composite hydrogels can always maintain a low value of $\sim 0.05$ under a high load of $5 \mathrm{~N}$ in the whole 10,000 test cycles (Fig. 7(b)). Correspondingly, the real-time friction coefficient 

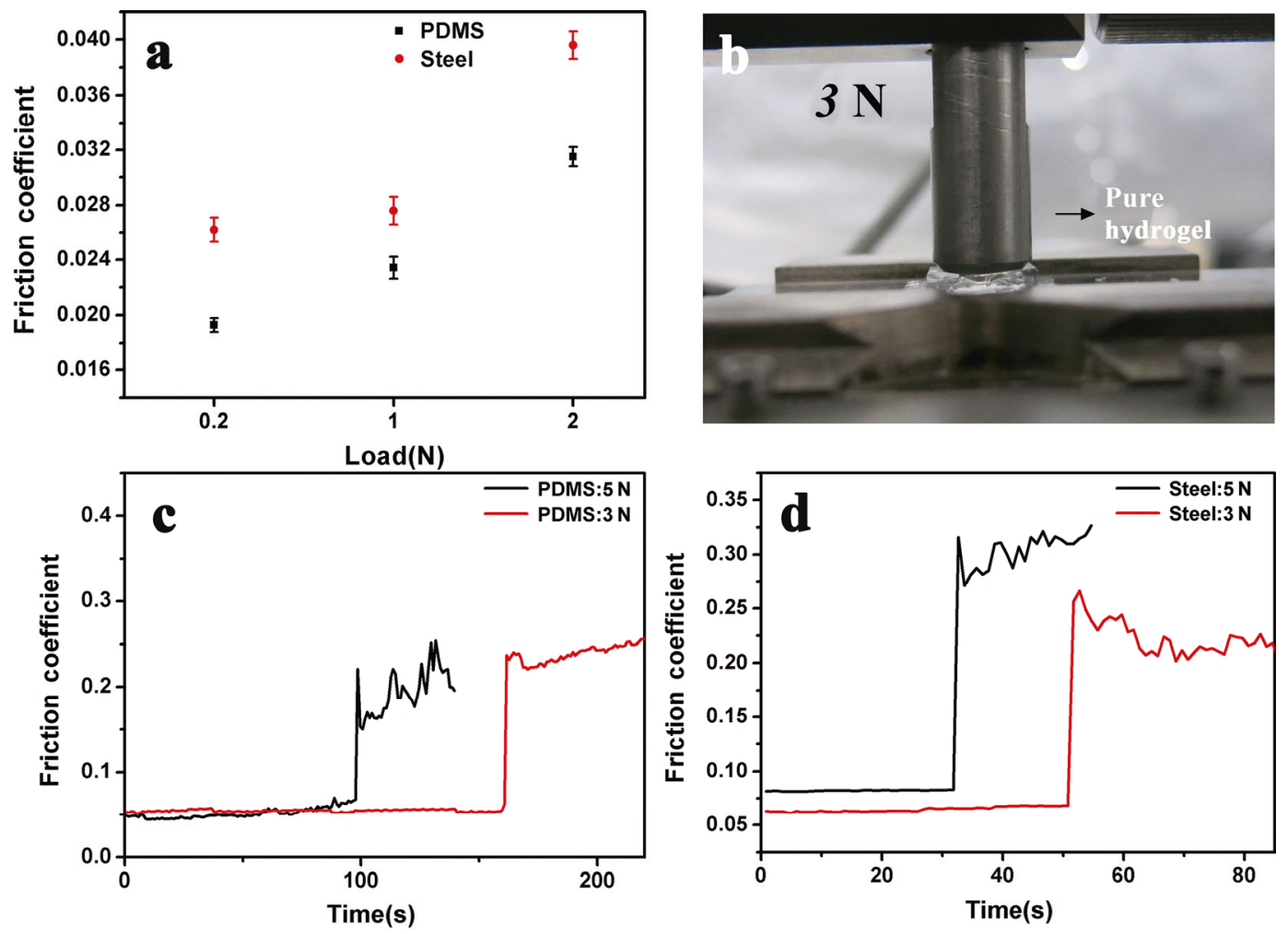

Fig. 6 (a) Change of friction coefficient values with normal loads upon employing PDMS ball and steel ball for pure PVP-PHEMA hydrogels; (b) the photograph of the local contact of pure PVP-PHEMA hydrogels against PDMS ball under normal load of $3 \mathrm{~N}$; the real-time friction coefficient curves of pure PVP-PHEMA hydrogels against (c) PDMS ball and (d) steel ball with sliding time under normal loads of 3 and $5 \mathrm{~N}$ (velocity: $0.01 \mathrm{~m} / \mathrm{s}$ ).

curves of spunlace fiber composite hydrogels against (Fig. 7(c)) PDMS ball and (Fig. 7(d)) steel ball were also provided. Compared with soft PDMS ball, the friction coefficient curves of the steel ball case generated slight fluctuations with the typical stickslip phenomenon. The reason responsible for this can be attributed to that hard steel pair would generate a higher contact stress to induce larger elastic deformation of hydrogel network under the same normal load, causing a much larger friction coefficient than that of using PDMS pair. Besides, to evaluate the wear-resistance capability of the composite hydrogels, the surface wear morphology of spunlace fiber composite hydrogels was also observed after encountering 10,000 reciprocating sliding cycles. As shown in Fig. 7(e), the surface of spunlace fiber composite hydrogels only appeared slight wear marks without generating obvious peeling, indicating its excellent load-bearing and anti-resistance capacities. As speculated, the spunlace fibers are tightly distributed within the hydrogels network based on the hydrogen bonding interaction, which would significantly improve the inherent robustness of the hydrogel network. Combining with the analysis for friction performance, these above results indicate that the as-prepared spunlace fiber composite hydrogels possess good lubrication, load-bearing, and anti-resistance properties.

Furthermore, the tribological properties of alginate fibers and cotton composite hydrogels were also investigated under different normal loads based on the same test condition. As shown in Fig. 8(a), the friction coefficients of the alginate fiber composite hydrogels are both significantly higher ( 0.135) than that of the spunlace fiber composite hydrogels when the PDMS ball and steel ball were used as the friction pairs (Fig. 7(a)). This indicates that the alginate fiber composite hydrogels would have poor wear-resistance property under high load condition, and its surface would rapidly reach the heavy-wear stage. This can be attributed to the fact that alginate fibers are too thin to perform a good load-bearing function (Fig. 2(b)). As shown in Fig. 8(b), the friction coefficient of the cotton 

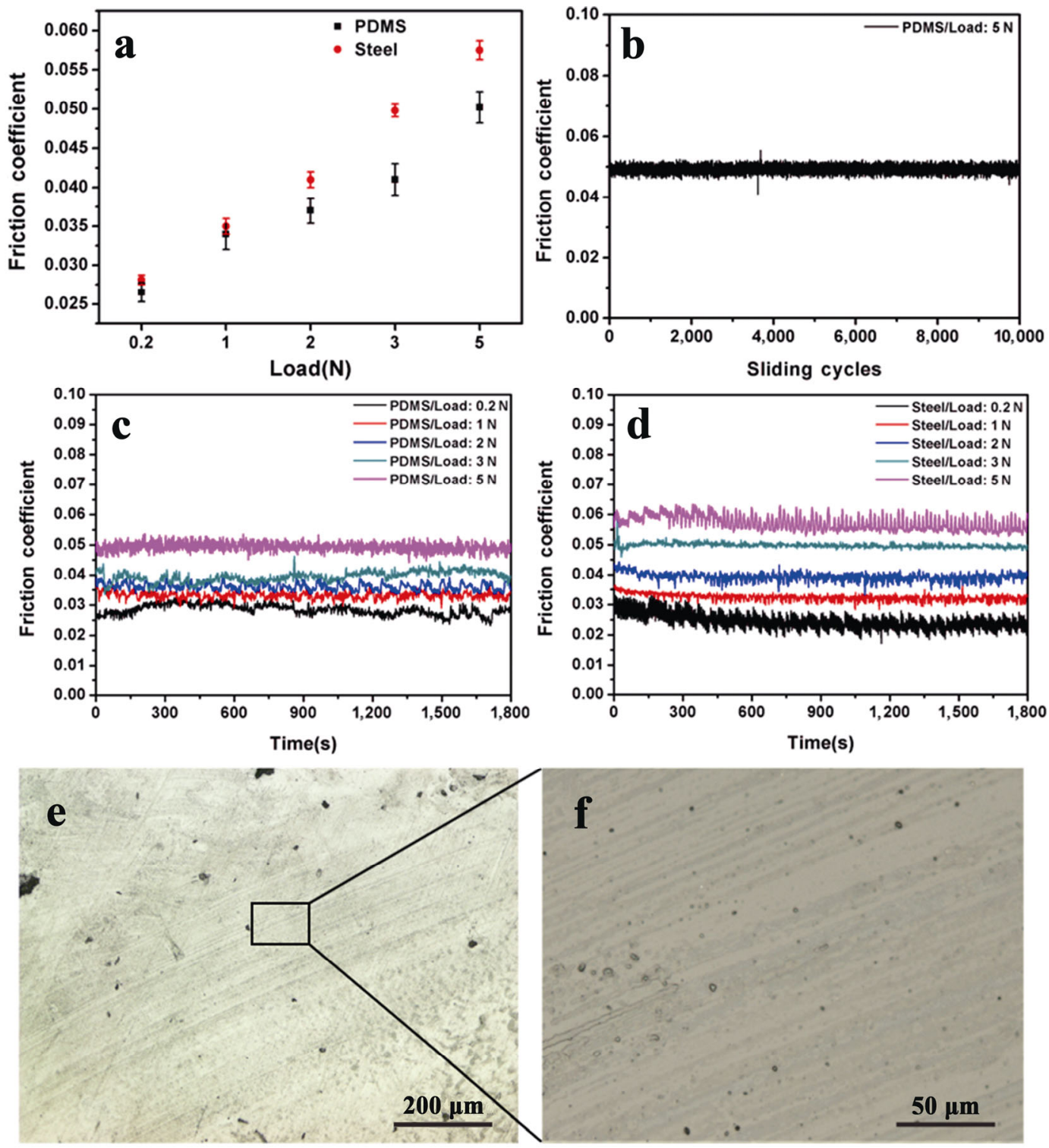

Fig. 7 (a) Comparison of friction coefficient obtained in both contact counterpart on spunlace fiber composite hydrogels at different normal loads; (b) the long-time friction coefficient curve with sliding time under the load of $5 \mathrm{~N}$ at the sliding velocity of $0.01 \mathrm{~m} / \mathrm{s}$ upon employing PDMS as friction pair; corresponding friction coefficient curves with sliding time under different loads when (c) PDMS and (d) steel ball were used as friction pairs; (e, f) the optical microscope images of the spunlace fiber composite hydrogels surface after encountering 10,000 friction tests cycles.

composite hydrogels is $\sim 0.115$ at the load of $0.2 \mathrm{~N}$ against PDMS pair and it increased to $\sim 0.15$ against the steel ball pair. This can be attributed to the loose fiber structure distribution inside the cotton (Fig. 2(c)), resulting in relatively rough surface after composition with the hydrogels. So, among these three composite hydrogels, the spunlace fiber composite hydrogels exhibit better tribological property than that of the other two composite hydrogels, which is highly related to its toughness and strength of fibers. However, for cotton fiber composite hydrogels, the fiber network is so dispersed along with poor toughness and strength to endure larger loads. As a result, the cotton composite hydrogels show relatively poor load-bearing and wear-resistance properties. By contrast, alginate fiber composite hydrogel shows moderate fiber distribution density, so its tribological property is between the spunlace fiber composite hydrogels and cotton fiber composite 

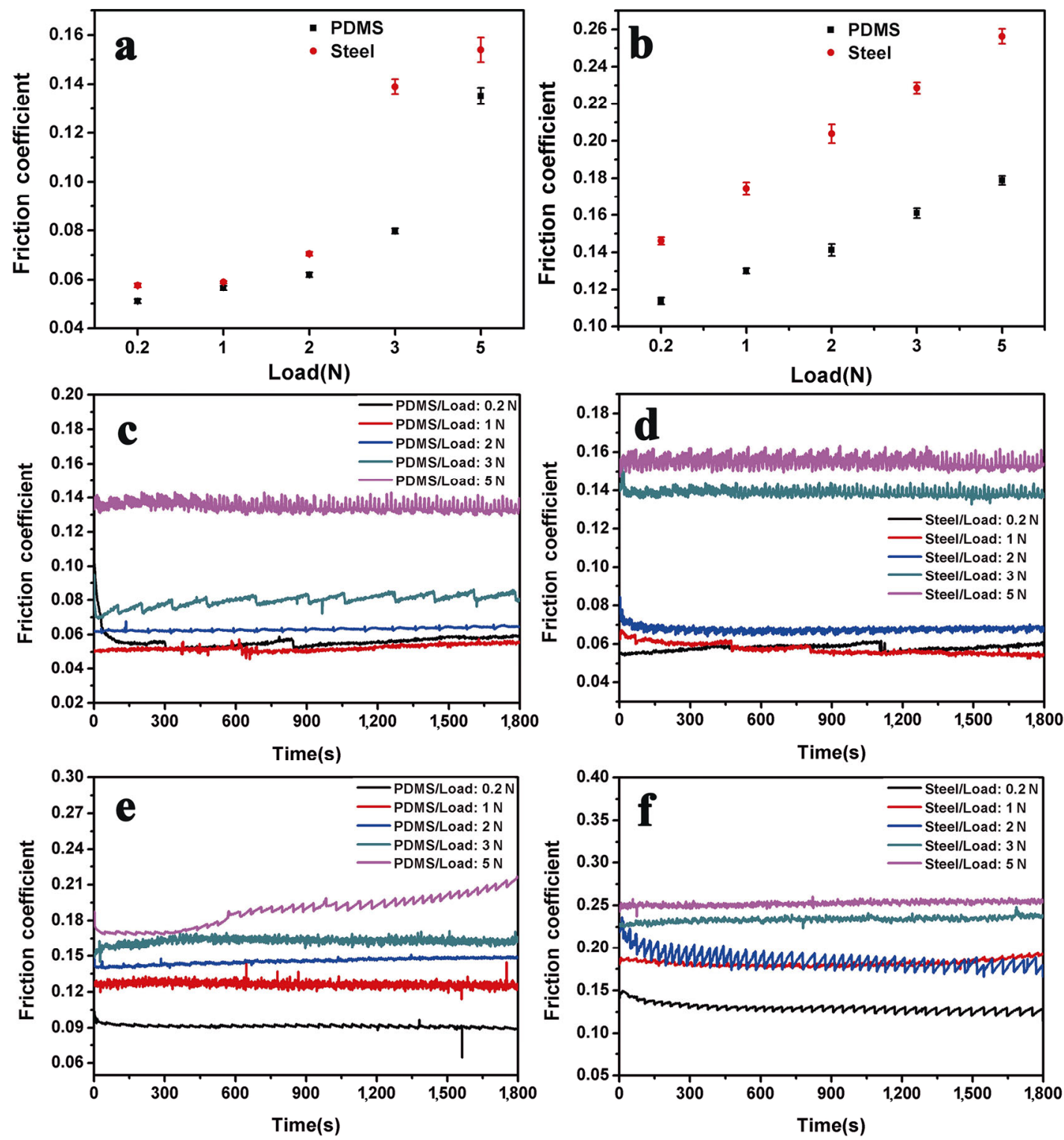

Fig. 8 Change of friction coefficient values with normal loads upon employing PDMS ball and steel ball as friction pairs for (a) alginate fiber composite hydrogels and (b) cotton composite hydrogels; typical friction coefficient curves with sliding time under different loads for alginate fiber composite hydrogels when (c) PDMS and (d) steel ball were used as friction pairs; typical friction coefficient curves with sliding time under different loads for cotton composite hydrogels when (e) PDMS and (f) steel ball were used as friction pairs.

hydrogels. In short, the combination of fibers and PVP-PHEMA hydrogels can satisfy the fundamental requirements of cartilage-like high strength and load-bearing.

\section{Conclusions}

Inspired by the structural feature of the articular cartilage network, several kinds of composite hydrogel materials are prepared by integrating fibers into hydrogel networks based on the hydrogen bond interactions. Compared with pure hydrogels samples, the fiber-enhanced composite hydrogels exhibit excellent mechanical properties. When the fiberenhanced composite hydrogels are performed with mechanical shearing and tearing, the fibers confined into the hydrogel network can dissipate energy by elongating the fracture, and thus highly enhancing the strength and toughness of the hydrogels. As a result, the wear-resistance and load-bearing properties 
of the composite hydrogels improve obviously. Taking the spunlace fiber-enhanced composite hydrogels as an example, it still shows stable lowfriction, excellent load-bearing, and wear-resistance after 10,000 cycles of sliding test using PDMS ball as friction pair. Meanwhile, it is found that the wear resistance of the as-prepared composite hydrogels is highly related to the properties of the fibers themselves. Overall, the combination of fibers and PVP-PHEMA hydrogels can successfully prepare the novel fiber-enhanced composite hydrogel materials with considerable mechanical strength and toughness. The composite hydrogels exhibit improved lubrication, load-bearing, and anti-wear properties in comparison with the traditional hydrogel system, and show considerable potential in the field of biomimetic materials.

\section{Acknowledgements}

The authors gratefully acknowledge the financial support from the National Natural Science Foundation of China (51705507, 51805514, and 51828302) and the Young Elite Scientists Sponsor Ship Program by China Association for Science and Technology (CAST) (2017QNRC0181), External Cooperation Program of Chinese Academy of Sciences (121B62KYSB20170009), and Key research projects of Frontier Science of Chinese Academy of Sciences (QYZDY-SSWJSC013).

Open Access This article is licensed under a Creative Commons Attribution 4.0 International License, which permits use, sharing, adaptation, distribution and reproduction in any medium or format, as long as you give appropriate credit to the original author(s) and the source, provide a link to the Creative Commons licence, and indicate if changes were made.

The images or other third party material in this article are included in the article's Creative Commons licence, unless indicated otherwise in a credit line to the material. If material is not included in the article's Creative Commons licence and your intended use is not permitted by statutory regulation or exceeds the permitted use, you will need to obtain permission directly from the copyright holder.

To view a copy of this licence, visit http:// creativecommons.org/licenses/by/4.0/.

\section{References}

[1] Sun T L, Kurokawa T, Kuroda S, Ihsan A B, Akasaki T, Sato K, Haque M A, Nakajima T, Gong J P. Physical hydrogels composed of polyampholytes demonstrate high toughness and viscoelasticity. Nat Mater 12(10): 932-937 (2013)

[2] Lee K Y, Mooney D J. Hydrogels for tissue engineering. Chem Rev 101(7): 1869-1880 (2001)

[3] Liao I C, Moutos F T, Estes B T, Zhao X H, Guilak F. Composite three-dimensional woven scaffolds with interpenetrating network hydrogels to create functional synthetic articular cartilage. Adv Funct Mater 23(47): 5833-5839 (2013)

[4] Ma S H, Yu B, Pei X W, Zhou F. Structural hydrogels. Polymer 98: 516-535 (2016)

[5] Pan Y S, Xiong D S. Friction properties of Nanohydroxyapatite reinforced poly(vinyl alcohol) gel composites as an articular cartilage. Wear 266(7-8): 699-703 (2009)

[6] Liu G Q, Cai M R, Feng Y G, Wang X L, Zhou F, Liu W M. Photothermally actuated interfacial hydration for fast friction switch on hydrophilic polymer brush modified PDMS sheet incorporated with $\mathrm{Fe}_{3} \mathrm{O}_{4}$ nanoparticles. Chem Commun 52(18): 3681-3683 (2016)

[7] Liu G Q, Cai M R, Wang X L, Zhou F, Liu W M. Magnetite-loaded thermosensitive nanogels for bioinspired lubrication and multimodal friction control. ACS Macro Lett 5(1): 144-148 (2016)

[8] Liu G Q, Liu Z L, Li N, Wang X L, Zhou F, Liu W M. Hairy polyelectrolyte brushes-grafted thermosensitive microgels as artificial synovial fluid for simultaneous biomimetic lubrication and arthritis treatment. ACS Appl Mater Interfaces 6(22): 20452-20463 (2014)

[9] Liu G Q, Wang X L, Zhou F, Liu W M. Tuning the tribological property with thermal sensitive microgels for aqueous lubrication. ACS Appl Mater Interfaces 5(21): 10842-10852 (2013)

[10] Gong J P, Kurokawa T, Narita T, Kagata G, Osada Y, Nishimura G, Kinjo M. Synthesis of hydrogels with extremely low surface friction. J Am Chem Soc 123(23): 5582-5583 (2001)

[11] Lin P, Zhang R, Wang X L, Cai M R, Yang J, Yu B, Zhou F. Articular cartilage inspired bilayer tough hydrogel prepared by interfacial modulated polymerization showing excellent combination of high 
load-bearing and low friction performance. ACS Macro Lett 5(11): 1191-1195 (2016)

[12] Wegst U G K, Bai H, Saiz E, Tomsia A P, Ritchie R O. Bioinspired structural materials. Nat Mater 14(1): 23-36 (2015)

[13] Moutos F T, Freed L E, Guilak F. A biomimetic threedimensional woven composite scaffold for functional tissue engineering of cartilage. Nat Mater 6(2): 162-167 (2007)

[14] Covert R J, Ott R D, Ku D N. Friction characteristics of a potential articular cartilage biomaterial. Wear $\mathbf{2 5 5}$ (7-12): 1064-1068 (2003)

[15] Hu X B, Vatankhah-Varnoosfaderani M, Zhou J, Li Q X, Sheiko S S. Weak hydrogen bonding enables hard, strong, tough, and elastic hydrogels. Adv Mater 27(43): 6899-6905 (2015)

[16] Li J Y, Suo Z G, Vlassak J J. Stiff, strong, and tough hydrogels with good chemical stability. J Mater Chem B 2(39): 6708-6713 (2014)

[17] Zhao D, Huang J C, Zhong Y, Li K, Zhang L N, Cai J. High-strength and high-toughness double-cross-linked cellulose hydrogels: A new strategy using sequential chemical and physical cross-linking. Adv Funct Mater 26(34): 6279-6287 (2016)

[18] Kaneko D, Tada T, Kurokawa T, Gong J P, Osada Y. Mechanically strong hydrogels with ultra-low frictional coefficients. Adv Mater 17(5): 535-538 (2005)

[19] Yi X, He J P, Wang X L, Zhang Y, Tan G X, Zhou Z N, Chen J Q, Chen D F, Wang R X, Tian W, et al. Tunable mechanical, antibacterial, and cytocompatible hydrogels based on a functionalized dual network of metal coordination bonds and covalent crosslinking. ACS Appl Mater Interfaces 10(7): 6190-6198 (2018)

[20] Fan C J, Liao L Q, Zhang C, Liu L J. A tough double network hydrogel for cartilage tissue engineering. $J$ Mater Chem B 1(34): 4251-4258 (2013)

[21] Xiang Y Q, Peng Z Q, Chen D J. A new polymer/clay Nano-composite hydrogel with improved response rate and tensile mechanical properties. Eur Polym J 42(9): 2125-2132 (2006)

[22] Abraham S, Brahim S, Ishihara K, Guiseppi-Elie A. Molecularly engineered p(HEMA)-based hydrogels for implant biochip biocompatibility. Biomaterials 26(23): 4767-4778 (2005)

[23] Li X Y, Zhang Z L, Chen H. Development and evaluation of fast forming nano-composite hydrogel for ocular delivery of diclofenac. Int $J$ Pharm 448(1): 96-100 (2013)

[24] Chen C, Dong A J, Yang J, Deng L D. Preparation and properties of an injectable thermo-sensitive double crosslinking hydrogel based on thiolated chitosan/beta- glycerophosphate. J Mater Sci 47(5): 2509-2517 (2012)

[25] Lin P, Ma S H, Wang X L, Zhou F. Molecularly engineered dual-crosslinked hydrogel with ultrahigh mechanical strength, toughness, and good self-recovery. Adv Mater 27(12): 2054-2059 (2015)

[26] Huang Y W, King D R, Cui W, Sun T L, Guo H L, Kurokawa T, Brown H R, Hui C Y, Gong J P. Superior fracture resistance of fiber reinforced polyampholyte hydrogels achieved by extraordinarily large energydissipative process zones. J Mater Chem A 7(22): 13431-13440 (2019)

[27] Huang Y W, King D R, Sun T L, Nonoyama T, Kurokawa T, Nakajima T, Gong J P. Energy-dissipative matrices enable synergistic toughening in fiber reinforced soft composites. Adv Funct Mater 27(9): 1605350 (2017)

[28] King D R, Sun T L, Huang Y W, Kurokawa T, Nonoyama T, Crosby A J, Gong J P. Extremely tough composites from fabric reinforced polyampholyte hydrogels. Mater Horiz 2(6): 584-591 (2015)

[29] Ribeiro A, Veiga F, Santos D, Torres-Labandeira J J, Concheiro A, Alvarez-Lorenzo C. Bioinspired imprinted PHEMA-hydrogels for ocular delivery of carbonic anhydrase inhibitor drugs. Biomacromolecules 12(3): 701-709 (2011)

[30] Huang J J, Liu G, Song C Y, Saiz E, Tomsia A P. Role of molecular chemistry of degradable pHEMA hydrogels in three-dimensional biomimetic mineralization. Chem Mater 24(7): 1331-1337 (2012)

[31] Long R, Hui C Y. Fracture toughness of hydrogels: measurement and interpretation. Soft Matter 12(39): 8069-8086 (2016)

[32] Bavaresco V P, Zavaglia C A C, Reis M C, Gomes J R. Study on the tribological properties of pHEMA hydrogels for use in artificial articular cartilage. Wear 265(3-4): 269-277 (2008)

[33] Young C D, Wu J R, Tsou T L. High-strength, ultra-thin and fiber-reinforced pHEMA artificial skin. Biomaterials 19(19): 1745-1752 (1998)

[34] Casadio Y S, Brown D H, Chirila T V, Kraatz H B, Baker M V. Biodegradation of Poly(2-hydroxyethyl methacrylate) (PHEMA) and Poly\{(2-hydroxyethyl methacrylate)-co-[poly(ethylene glycol) methyl ether methacrylate]\} Hydrogels Containing Peptide-Based Cross-Linking Agents. Biomacromolecules 11(11): 2949-2959 (2010)

[35] Marambio O G, Pizarro G D C, Jeria-Orell M, Geckeler $\mathrm{K}$ E. Swelling behavior and metal ion retention from aqueous solution of hydrogels based on N-1-vinyl-2pyrrolidone and $N$-hydroxymethylacrylamide. $J$ Appl Polym Sci 113(3): 1792-1802 (2009) 


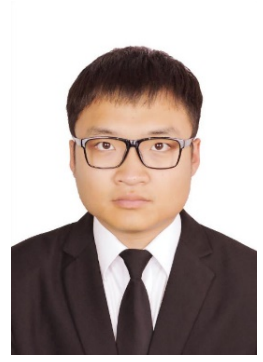

Jiawei LI. He obtained his bachelor degree in Hunan University of Technology, China (2015). He got his master degree at Lanzhou Jiaotong University, China, 2019. He studied about one year (2018-

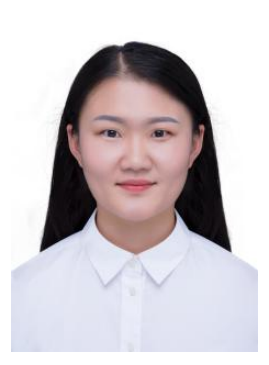

Luyao GAO. She obtained her bachelor degree in 2016 from Ningxia University. She is now a Ph.D. candidate in LICP. Her current

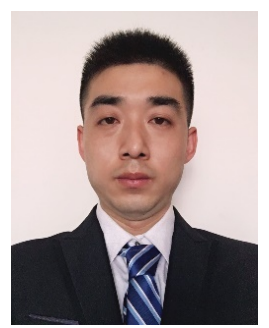

Shuanhong MA. He received his bachelor degree in Tianshui Normal University, China (2011). He got a Ph.D. degree in LICP, CAS (2016). Now, he is an associate professor at the State Key Laboratory of Solid Lubrication, LICP, CAS. He has published over 55 papers, obtained more than 20 patents for invention, and received nearly 1,100 citations. He is a senior member of the Chinese

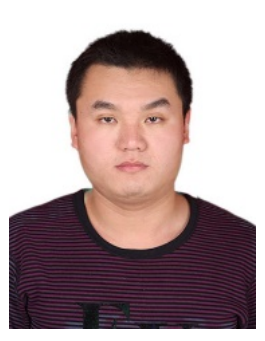

Yang WU. He received his bachelor degree in Qufu Normal University, China (2010), and his Ph.D. degree at LICP, CAS, 2015. He has been a research assistant at City University of Hong Kong (2016-2017). He has published 25 papers (e.g., Nat Commun) and obtained more than
2019) at the State Key Laboratory of Solid Lubrication, Lanzhou Institute of Chemical Physics (LICP), Chinese Academy of Sciences (CAS), as a joint training student. His research interest is the tribological properties of soft matters-based composite materials.

research interests mainly focus on organic synthesis of biomacromolecules and polymers-based wet/ adhesion/lubrication materials.

Mechanical Engineering Society (CMES) and the host of the Young Elite Scientists Sponsorship Program of CAST (2017QNRC0181). He is one of the main inventors for surface catalytic initiated radical polymerization (SCIRP) method in the world. His current research interests focus on the surface wet/lubricious modification, biolubrication and mechanical bionic tribology (basic research), and product development of lubricious materials \& medical devices (application technology).

10 patents for invention. Now, he is an associate professor at the State Key Laboratory of Solid Lubrication, LICP, CAS. His research interests mainly focus on the interface friction control, aqueous lubrication, and underwater adhesion of soft materials (basic research), and product development of low surface energy \& lubricious coatings (application technology). 